\title{
The City as a Learning Gamified Platform
}

\author{
Aldo Gordillo, Daniel Gallego, Enrique Barra and Juan Quemada \\ Escuela Técnica Superior de Ingenieros de Telecomunicación \\ Universidad Politécnica de Madrid \\ Avenida Complutense 30, 28040, Madrid, Spain \\ \{agordillo, dgallego, ebarra, jquemada\}@dit.upm.es
}

\begin{abstract}
The area of mobile city guides has grown really fast in the last years based on new mobile capabilities. This growth has been fostered by the evolution of ubiquitous systems and the great penetration of smartphones in the society. In this paper we propose a generic model to support a new way of visiting the city: instead of as a place for tourism, we see it as a place for learning in which located educational resources are available for end users. The model has been conceived as a way to encourage them to create their own educational tours, in which Learning Points Of Interest are set up to be discovered. Two main use cases are supported by the model: formal (conducted by a teacher) and informal (no educator is related to the learning experience) outdoor mobile learning. Details about the impact of the conjunction of tourism, learning and gamification dimensions in the model design, as well as about the model itself are provided. Finally, a mobile application prototype developed in the context of the FI-CONTENT European project is presented as a proof of concept of the model.
\end{abstract}

Keywords-Mobile Learning; Gamification; Educational Tours; Learning Object

\section{INTRODUCTION}

The area of mobile city guides has grown really fast in the last years based on new mobile capabilities such as connectivity or location context. This growth has been fostered by the evolution of ubiquitous systems and the great penetration of smartphones in the society. Mobile city guides are changing the way people experience tourism, plan their travels and share these experiences. Many mobile city guides exist nowadays such as Stay [1], Tourist Eye [2], or Lonely Planet [3] providing guides for travelling worldwide. Moreover, a lot of cities offer also their own mobile city guide. The main aim of these guides is to provide basic information about locations that are useful or interesting. These locations are formally known as Points Of Interest (POIs). Other common features are personalized recommendations, customized travel guides, sharing travelling information, offline access and augmented reality functionalities. In addition, new features can be added to these mobile tourist applications in order to provide new services. For instance, [4] proposed a mobile tourist service built by integrating mobile guide services with other services such as notifications and payments. In the same way, these mobile applications can be expanded further to include mobile learning. So, they can be repurposed in the education domain. Bearing in mind this idea, we propose a new way of visiting the city: instead of a place for tourism we see it as a place for learning in which located educational resources are available for end users interested in discovering history, legends or curiosities about the cities they live in or visit. Furthermore, in order to provide engaging and motivating learning experiences a gamified dimension is also considered together with the tourism and learning dimensions.

The idea of enriching and gamifying traditional tourist tours is not new. For instance, the "Matera Tales of a City" project [5] provides a web platform and a gamified mobile application to allow tourists to access to cultural contents (e.g. 3D reconstructions or audio guides) while attending places.

Using a real city (or the world) as a gamified scenario is not new either. The stage used to play MEECO [6] is a real city, where citizens can virtually register their real ecological actions such as recycle or biking using their mobile devices.

Nevertheless, no learning approaches have been defined to take advantage of mobile city guides benefits in the educational field. Bearing this in mind, we present in this paper a generic model that defines a new learning approach based on the use of the city as a learning gamified platform considering three dimensions: tourism, learning and gamification. This new learning approach arises from the combination of the areas of mobile city guides, mobile learning and gamification according to Fig. 1.

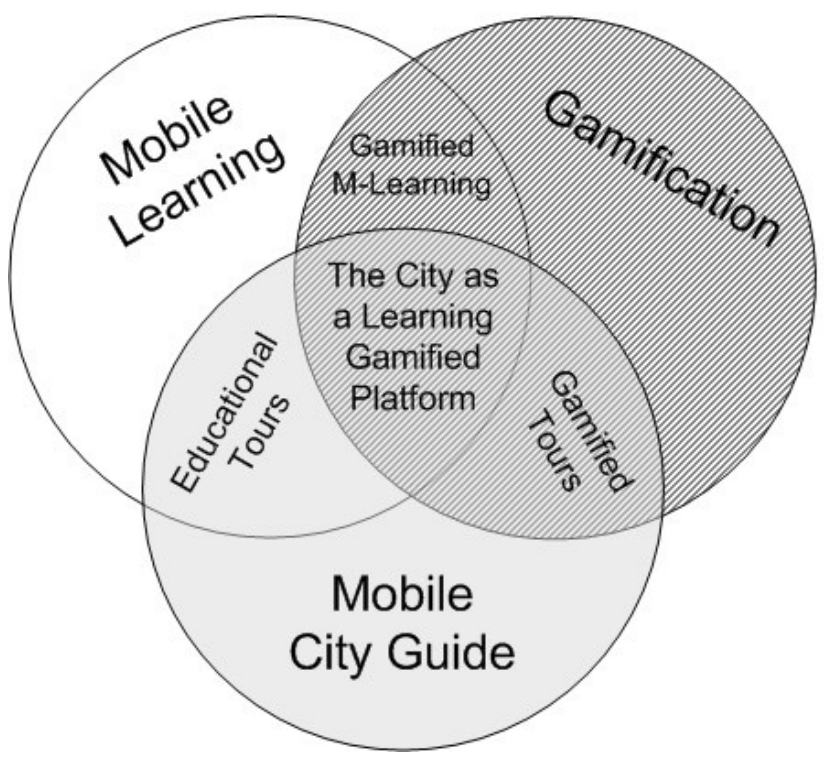

Fig. 1. The City as a Learning Gamified Platform research field. 
The model has been defined based on a scenario designed in the context of the FI-CONTENT (http://www.fi-content.eu) European project. One of the aims of this project is to validate and evaluate the service infrastructure provided by the FI-WARE (http://www.fi-ware.eu) European project. For this reason, a scenario based on the city as a gamified platform was proposed and selected to test specific components and technologies developed as part of the FI-WARE project. The main use cases and requirements of this scenario were identified and used as a basis for the model definition. Lastly, as a proof of concept, an application prototype has been developed using FI-WARE elements.

The rest of the paper is organized as follows. The next section reviews related work of mobile learning, adventure in education and gamification. Section 3 introduces the FI-CONTENT and FI-WARE projects and illustrates the main use cases. Section 4 explains the model in detail. Section 5 presents the application prototype. Then, section 6 provides some discussion. The last section finishes with some concluding remarks together with an outlook on future work.

\section{RELATED WORK}

As technology evolves, faster wireless and mobile networks and more powerful handheld devices are available at lower cost. These technological advancements are creating the ideal conditions for Mobile Learning (M-Learning). There are several definitions of the term M-Learning. A commonly accepted one can be found in [7] which defines M-Learning as "any sort of learning that happens when the learner is not at a fixed, predetermined location, or learning that happens when the learner takes advantage of the learning opportunities offered by mobile technologies". This definition can be completed by adding that M-Learning also aims to allow learners to assimilate learning anywhere and at any time [7], [8]. Another common goal of M-Learning applications is to allow learners to accomplish learning activities by taking all contextual elements into consideration [9]. This brings the concept of context. According to [10] context is "any information that can be used to characterize the situation of an entity", where an entity is defined as "a person, place or object that is considered relevant to the interaction between a user and an application, including the user and applications themselves". Some studies have already showed that learning can be effectively extended to handheld devices and that M-Learning applications can be used to complement classroom or distance learning [11]. This type of learning opens up new opportunities for educators because the learning activity is not limited to the classroom; it can also be completed anywhere and anytime, without requiring strict supervision by the teacher [12]. Novel learning opportunities can also arise by introducing collaboration [13]. These new chances are especially relevant for informal learning, where the learning activities usually take place outside educational establishments and formal curricula of schools.

On the other hand, new opportunities in formal and informal learning can also be achieved by incorporating adventure into education. Some approaches aim to achieve this goal in a pedagogically meaningful way. The first one was Adventure Education (AE), which is a form of experiential learning typically associated with cooperative games, problem-solving tasks and high adventure activities [14]. Despite AE not necessarily occurs out of the classroom, it is sometimes used wrongly as synonymous of outdoor learning, which is precisely characterized by experiencing learning activities in the outdoors. Another approach called Adventure Learning (AL) was defined by Doering in 2006 [15]. It can be defined as a hybrid distance education approach that provides students with opportunities to explore real-world issues through authentic, field-based narratives within an online learning environment. Lastly, one more approach exists based on the novel concept of User-Drive Adventure Learning Environments (UDALE) allowing learners to create and share self-initiated AL projects. UDALE merges components of AE and AL. It aims to engage learners in their own physical adventure while concurrently teaching others about a real-world issue, sharing authentic data and employing technology for data collection and collaboration [16]. Due to UDALE tools, mobile learning is being explored as a mean to introduce adventure into education and engage learners in AL.

Finally, educators can also provide new learning experiences by applying gamification in education. Although gamification lacks a universal definition an appropriate one can be found in [17], which defines gamification as "the use of game design elements in non-game contexts". In education, gamification can be used to promote learning because many of the elements of gamification are based on educational psychology and are techniques that designers of instruction, teachers, and professors have been using for years [18]. Examples of common elements used in gamified applications are, among others, badges, leaderboards and scoring systems. A typical example of a gamified application is Foursquare (http://www.foursquare.com), in which users can collect badges for accomplishing activities such as check-in a certain number of times to specific POIs (e.g. 20 different pizza places). Hence, gamification can be applied to M-Learning to provide more engaging learning experiences, especially beyond the classroom walls.

\section{SCENARIO}

\section{A. FI-CONTENT and FI-WARE projects}

FI-WARE is a European project which main goal is to advance the global competitiveness of the European Union economy by introducing an innovative infrastructure for cost-effective creation and delivery of versatile digital services, providing high QoS (Quality of Service) and security guarantees. The project also intends to demonstrate how this infrastructure can support novel Future Internet (FI) services in multiple sectors such as healthcare, telecommunications or education in a more productive, reliable and efficient way. The service infrastructure is built upon building blocks called Generic Enablers (GEs). A GE can be defined as a component that offers at least one reusable and commonly shared functionality and can serve a multiplicity of usage areas. The identification and specification of GEs are key goals of the FI-WARE project together with the development of reference implementations of them. 
The FI-CONTENT European project aims to shape the next generation Internet providing a framework to allow transformations of novel and inventive scenarios into technical outputs and demonstrating usage beyond current state of the art. FI-CONTENT is focused on several content areas such as virtual environments, games, educational entertainment and culture and user created content. The scenarios are proposed by a consortium formed mostly by major content providers, game companies, broadcasters, research institutes and academic institutions. One of the foremost aims of FI-CONTENT is to validate and evaluate the existing GEs provided by FI-WARE in different scenarios for their future implementation by using working prototypes.

\section{B. Use cases}

A scenario grounded on the city as a gamified platform can provide several novel learning experiences. These experiences are very diverse but all of them share the mobility component. Learners are moving across the city visiting different places, i.e. the learning activities are undertaken outdoors. All of these activities will interact in some respects with different elements of the city such as citizens or locations. On the one hand, learning can be produced by visiting POIs and gamification can be used to encourage participants to do that. On the other hand, people can interact in different ways collaborating, competing, teaching or leading. Taken into account that the presence of an instructor influences significantly the learning experiences in this scenario, two main use cases were identified related to formal and informal outdoor learning, respectively.

\section{1) Formal Outdoor Learning}

This use case describes a scenario in which a teacher creates an educational tour targeted for his/her students related to the current subject that is being studied in class. First off, the teacher creates the educational tour selecting the different POIs and learning materials. Besides adding existing materials, the teacher can create new specific contents or adapt the existing ones. Then, the teacher has to explain the rules of the learning experience in the classroom. Students can use their own smartphones to access to the educational tour and perform all the activities available. The tour starts leaving the classroom and adopting the entire city as the learning environment. Since this moment, students are involved in an adventure in which they have to achieve a set of goals. The achievement of the goals is based on the discovery of selected city locations and the realization of learning activities related to these places. These activities will be triggered when the students arrive to the associated location. A learning activity can be, for instance, watching a video about the place, a quiz that asks questions that require the student to explore physically the place to answer or a puzzle game that challenges the student's intelligence. Moreover, several elements may be involved in an activity. For instance, a learning activity may consist of a video and a quiz that ask a question about it. This way, students have to overcome diverse challenges based on the realization of learning activities through their mobile devices. As a consequence of performing successfully a learning activity, new goals or missions can be offered to the student and new elements can come into play. Finally, other actions can be carried out by the students during the tour such as take geo-located photos about specific topics and share them through an online environment.

\section{2) Informal Outdoor Learning}

This use case describes a learning scenario which lacks the presence of an instructor and where anyone can create his/her own educational tour and offer it to the community. The learning experience is based on the same principles than the previous use case. Each participant has to achieve a set of goals, whose achievement depends on the discovery of POIs and the realization of specific activities. However, this use case is focused on providing more joyful and engaging learning experiences in the process of discovering POIs. First off, each participant or player assumes the role of a character like in a role playing game. Optionally, the adventure can be set in a fictional or historical setting, explaining the backstory and the motivation. Each character has its own mission composed by a set of goals. In this scenario, Virtual Objects (VOs) are scattered around the city. Players can find VOs in some POIs, and they can watch and collect them via their mobile devices using augmented reality. Players can also trade with VOs. New goals are defined around VOs. For instance, the mission of a player may be to collect all of the pieces of the Philosopher's Stone and bring them to a specific spot. Some goals may also require collaboration to be achieved. This way, players are involved in an adventure which tests their ingenuity and their problem-solving and teamwork skills. Finally, two game elements are included in the scenario to encourage competition: leaderboards and badges. The leaderboard is built based on scores that can be earned by achieving goals or finding VOs, while badges can be collected undertaking different actions.

\section{Requirements}

Based on these use cases, several requirements were identified and numbered from Requirement 1 to Requirement 7.

1. The learning experiences must be provided by educational tours, which should be available for mobile devices since the learning occurs outdoors. Furthermore, they should be able to be created and designed by anyone.

2. Learning activities (e.g. videos, quizzes, mini games) are very diverse and can be combined to build more complex ones. Therefore a logical choice is to build them as Learning Objects (LOs) [19], [20].

3. The learning activities are tied to locations and hence the LOs must be associated with POIs.

4. Virtual Objects (VOs) are tied to POIs and participants can obtain them using their mobile phones.

5. Each participant may have a character associated with a specific mission and/or goals to achieve. Some goals may require teamwork to be achieved.

6. Several actions performed by participants or triggered by the occurrence of certain events have to be considered (e.g. reveal new POIs or goals, take a picture, etc.). 
7. Certain events (e.g. activity completion or POI discovery) should allow participants to achieve badges or earn a certain amount of score points. A leaderboard based on these scores ought to be provided.

\section{MODEL}

Based on the requirements derived from the use cases analysis, we define a model for the educational tour entity considering three dimensions: tourism, learning and gamification. This means that any educational tour can be represented by three components or vectors, each of them related to one dimension. The magnitude of these components relies on diverse factors of the educational tour such as LOs, POIs or VOs quantity, academic load or the role played by game mechanics in the learning experience. Therefore, educational tours tailored for different use cases will lead to different representations. To illustrate this idea, Fig. 2 shows the representation of the educational tours corresponding to the above mentioned use cases.

In the first case the learning component is the strongest one, since the educational tour has been created by a teacher with a strict educational purpose. Gamification is limited to provide a set of goals and maybe by including puzzle-like LOs. On the other hand, the second case has a very strong gamification component due to the preparation of the tour as a role playing game. In this case the LOs will be in most cases riddles, mini games, jigsaws and ability tests, which sometimes can offer less educational contents for the sake of entertainment and thus the learning component can be weaker. The tourism component mainly relies on the quantity of POIs. However, the more time participants spent consuming LOs, less time they will have to visit new locations.

\section{A. Educational Tour Structure}

In order to allow people to easily create educational tours regardless of their technical knowledge, they should use an e-Learning authoring tool. For this reason, the educational tours must follow a standardized structure that can be easily represented. This way, they can be implemented as web contents accessible through mobile devices and exported to standard e-Learning formats like SCORM (Sharable Content Object Reference Model) [21]. As a consequence we achieve the Requirement 1.

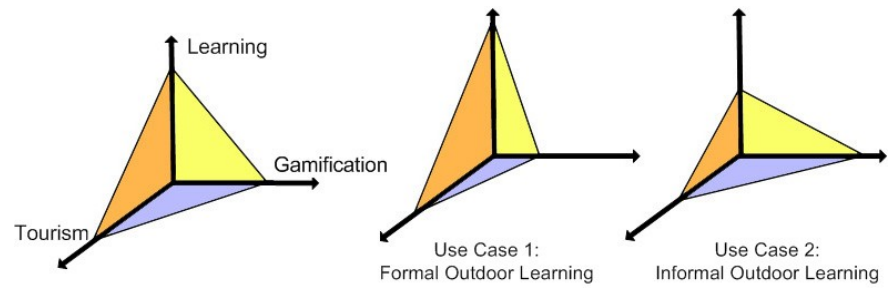

Fig. 2. Educational Tour Representation.
This model defines the structure of the educational tours including the description of the different components, their properties and how they relate among themselves. Fig. 3 summarizes this structure.

The learning activities are the building blocks of the tour. Providing them as LOs allows people to easily create and customize them conforming to e-Learning standards by using e-Learning authoring tools. Furthermore, it allows the reuse of a big amount of existing learning materials. Requirement 2 is met thanks to this strategy.

To associate the LOs with POIs (i.e. Requirement 3), we have created the concept of Learning Point of Interest (LPOI). A LPOI can be defined as a POI that has a LO associated, or in other words, a LO that is tied to a location. Since a LPOI is nothing more than a particular LO, it can be formed by aggregating other LOs. The set of all LPOIs is called scenario.

In an educational tour, a LPOI can also be associated with Virtual Objects (VOs). Requirement 4 is fulfilled as consequence of this linkage. In addition, VOs can be used by participants to touch off specific events in the tour (e.g. by bringing one to a specific POI).

An educational tour has a set of characters or roles. Each role has one mission with a uniquely associated goal and optionally a set of initial VOs. Goals are always related to LPOIs, VOs or both. The model considers three types of goals: go to a LPOI and accomplish the learning activities (i.e. consuming the LOs), bring a VO to a LPOI, and a combination of any of them. For instance, a collaborative goal can be defined by linking two simple goals: bring a VO to one location and bring another VO to a different location. Since a participant cannot be in two locations at the same time he/she will have to look for a partner. Since all of the goals are defined objectively based on existing entities, it is easy for a mobile application to manage them. Moreover, missions can also be split into activities. An activity is like a mission, but its associated goal is a "partial goal". This structure allows the achievement of the Requirement 5.

The accomplishment of an activity may result in the execution of an action. Actions cause effects over different entities of the tour. The main actions are: "Reveal a hidden LPOI", "Show a new activity" and "Give one VO to a certain participant". Actions can be performed as a response to a particular event (hence defined by the author of the tour), or because of a participant decision. For example, a VO can be given to a participant for discovering a LPOI or because of a barter deal between players. This modeling of the action entity covers the Requirement 6. 


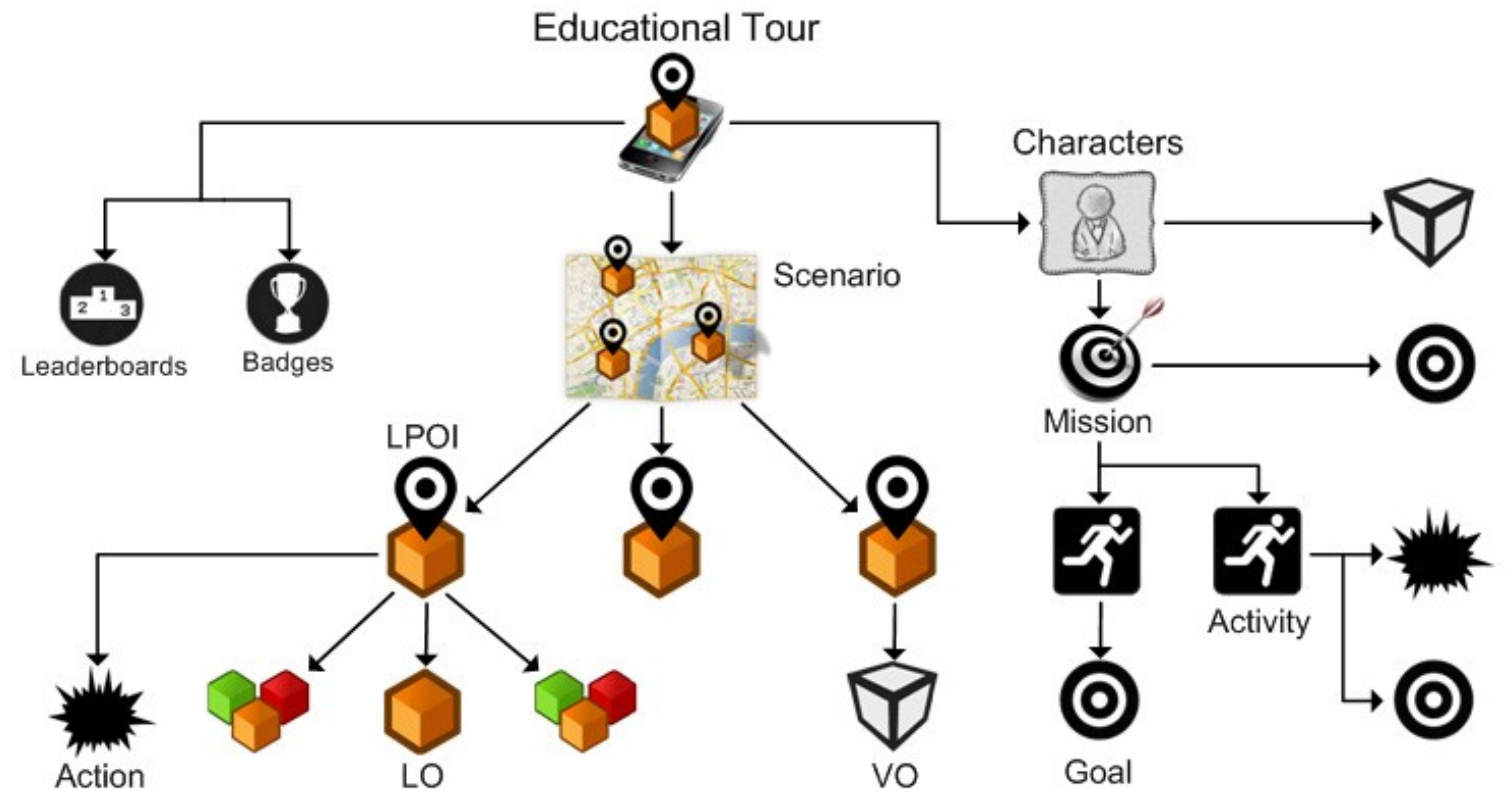

Fig. 3. Educational Tour Structure.

Lastly, in order to satisfy the Requirement 7, two more elements are considered in an educational tour: a leaderboard and a set of badges. There are two actions related to this: "Give a badge to a specific participant" and "Add score to a specific participant". Thereby, a participant can earn score points or achieving a badge by discovering and/or realizing the learning activities of a LPOI or by accomplishing a goal.

\section{PROTOTYPE}

As a proof of concept, an application prototype (Fig. 4) has been developed on top of the cloud services provided by the following GEs of the FI-WARE platform.

- Location to retrieve the users' location and notify the system when a user arrives to a LPOI.

- Publish/Subscribe Context Broker to notify users about certain news or events.

- Identity Management to provide authentication and authorization.

The application provides a set of educational tours of the city of Barcelona. Each tour is grounded on an historical character who will guide the user around the city. These characters explain different stories and curiosities related to their experiences throughout the tour. Characters can also propose different activities to accomplish. Fig. 4a shows the initial screen where a historical character from Barcelona can be chosen between Antoni Gaudi, Joan Miró or Pablo Picasso among others. After the selection of a character (in the Fig. 4 Antoni Gaudi) the educational tour starts. Then several LPOIs are revealed in a map as well as the current user's location. Fig. 4b represents this situation, where three LPOIs are showed: one in the Güell Park, other in the Sagrada Familia and another one in the Casa Batlló. Moreover, the user receives an initial advice from the virtual fellow traveller, recommending him/her to visit one LPOI. As we can see in Fig. 4b Antoni Gaudi is recommending the user to visit the Sagrada Familia. If the user follows this advice, a clue to perform activities related to the LPOI will be given upon his/her arrival. In the case illustrated, Gaudi confesses that he lost his diary in somewhere in the surroundings of the Sagrada Familia, encouraging the user to look for it. Fig. 4c shows the result of finding the VO representing the diary using augmented reality capabilities.

This application meets the two above mentioned use cases. This was achieved by providing several educational tours, each of them tailored for a different use case. On the one hand, it provides educational tours that could have been created by teachers with strong learning objectives related to the current subject studied in their classroom (e.g. an art history class). On the other hand, it also provides educational tours with weaker educational purposes, focusing more in the tourism and gamified dimensions. This kind of tours could have been created, for instance, by the tourism office of Barcelona or by a group of friends. 


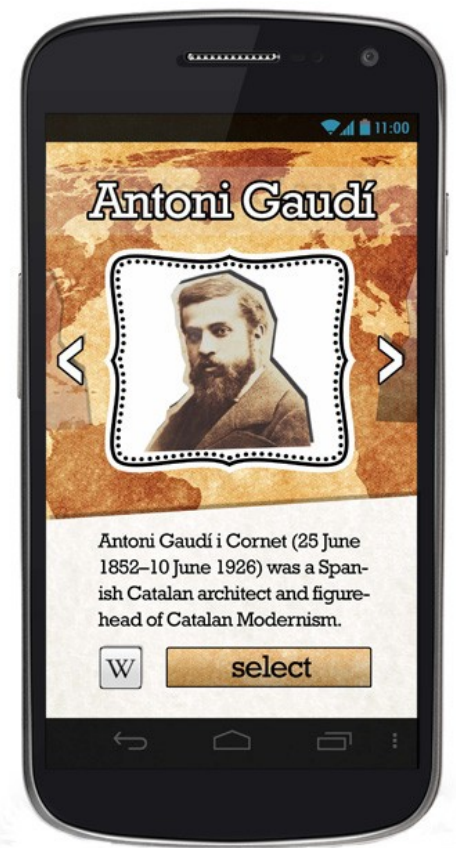

a)

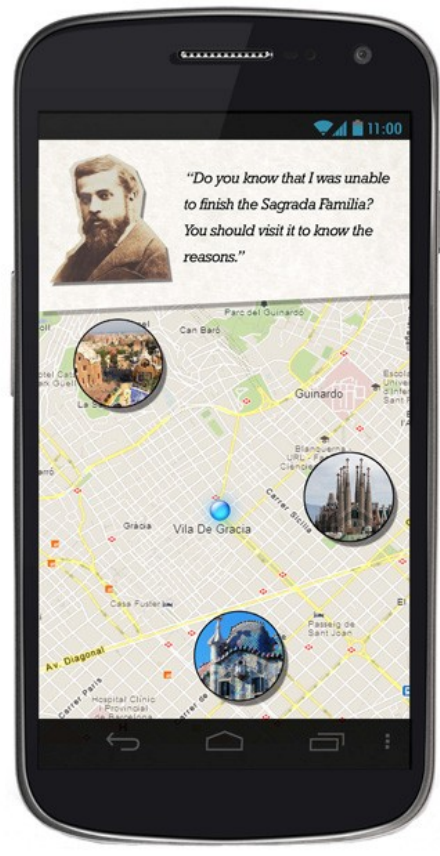

b)

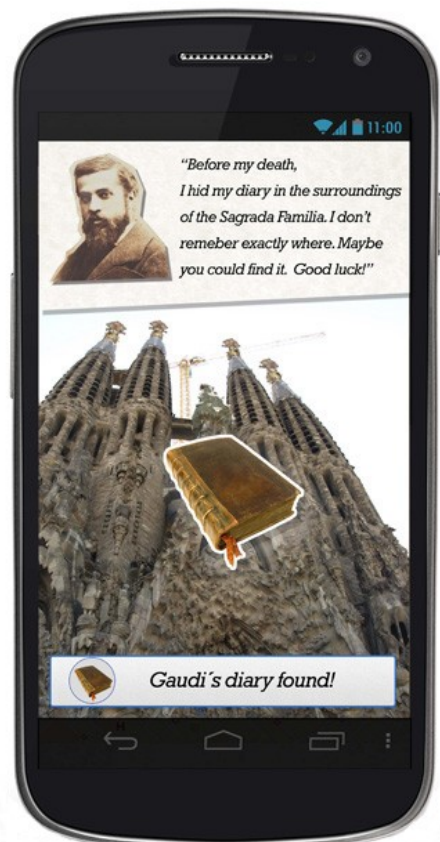

c)

Fig. 4. Prototype screenshots.

\section{DISCUSSION}

The model presented in this paper satisfies several use cases of outdoor M-Learning. Learning approaches focused on incorporating adventure into education such as $\mathrm{AE}$ and $\mathrm{AL}$ usually involves the realization of learning activities outdoors. Hence, in these cases, the model can be very useful providing more engaging learning experiences. Furthermore, the learning experiences can be tailored to be adapted to the desired use case by strengthening the correspondent component (tourism, learning or gamification). Another application of this model can be found in the emerging UDALE environments, since one of the challenges in its implementation is to provide an optimal structure and scaffolding for teachers and learners to allow them to create their own learning experiences [16].

However, some limitations exist when the model is put into practice. Learning experiences rely on mobile devices and hence they are limited by the technology capabilities of these devices. For example, if the mobile device is unable to determine the current location of the user for a prolonged time, they would not detect if the user arrives to a new LPOI. This fact can ruin the whole learning experience. Therefore, this technological issue limits the scope where the model can be applied. We designed the model for a city scenario, but it can fail or being unable to be applied to rural areas, where the connectivity is worse, or even to indoor scenarios where the geo-location is really complicated.

Finally, bearing in mind the tourism, learning and gamification components in which an educational tour can be decomposed, it is worth questioning if educational tours with a strongest learning component are the most effective or if it is better to include game elements to increase motivation at the expense of a weaker learning component.

\section{CONCLUSIONS AND FUTURE WORK}

We have presented a generic model which allows creating educational tours covering the main use cases of a scenario grounded on the city as a learning gamified platform. This model was created with the main aim of taking advantage of the benefits of linking the areas of Mobile City Guide, M-Learning and Gamification. As a consequence, the created educational tours can be represented by three dimensions: tourism, learning and gamification. Lastly, as a proof of concept, a mobile application prototype has been developed in the context of the FI-CONTENT European project to guarantee the achievement of the use cases and to ensure that the model is ready to be applied in real scenarios.

We have showed that the city can be effectively used as a place for learning. Several novel learning opportunities can be achieved in formal and informal outdoor M-Learning by using the city as a learning platform in combination with gamification. Also, this approach can enhance the experiences in existing learning approaches such as Adventure Education or Adventure Learning. Although these learning experiences can be limited by technological constraints, these limitations will be overcome since the technological advancements are creating the ideal conditions to use the city as a gamified learning platform.

Open challenges after this research consists of refining the mobile application prototype into a final product ready to be delivered to the public as well as the different components used in the service. Also, we plan to develop an authoring tool capable of creating the educational tours according to our model. This will allow us to test completely this new learning approach in the real world with teachers, learners and citizens as end users. 
Since this is a novel research field, it opens several new research lines towards the exploitation of the city as a learning environment. In the considered scenario, places are passive elements. Learners can interact with the Learning Objects associated with them but not with the places themselves. Thereby, an interesting research line to investigate would be to consider in the model that different places and urban elements may be intelligent entities that interact with the participants. For example, an intelligent recycle container could give a badge or score points to a participant (or citizen) for recycling appropriately a certain amount of trash. Bearing all this in mind, we believe that Smart Educational Cities can become a next frontier in education.

\section{ACKNOWLEDGMENT}

We wish to acknowledge our gratitude and appreciation to all the FI-WARE and FI-CONTENT project partners, and each one of the project team members, for their contribution during the development of various ideas and concepts presented in this paper. We also would like to thank the SAAN project (TIN2010-19138) for funding this work.

\section{REFERENCES}

[1] "Stay.com," 2013. [Online]. Available: http://www.stay.com.

[2] “Tourist Eye," 2013. [Online]. Available: www.touristeye.com.

[3] "Lonely Planet," 2013. [Online]. Available: http://www.lonelyplanet.com.

[4] C. Yu and H. Chang, "A Multi-facet Requirement Assessment of Customer-Oriented Mobile Tourism Services," in Proceedings of the Second International Conference on Next Generation Mobile Applications, Services, and Technologies (2008), 2008, pp. 263-268.

[5] E. Pietroni, "An augmented experiences in cultural heritage through mobile devices: 'Matera tales of a city' project," in Proceedings of the 18th International Conference on Virtual Systems and Multimedia (2012), 2012, pp. 117-124.

[6] D. Vara, E. Macías, S. Gracia, A. Torrents, and S. Lee, "MEECO: Gamifying ecology through a social networking plataform," in Proceedings of the 2011 IEEE International Conference on Multimedia and Expo, 2011.
[7] M. L. Crescente and D. Lee, "Critical issues of m-learning: design models, adoption processes, and future trends," Journal of the Chinese Institute of Industrial Engineers, vol. 28, no. 2, pp. 111-123, 2011.

[8] J. S. Kossen, "When e-learning becomes m-learning," Palmpower Magazine Enterprise Edition, 2001.

[9] A. Al-Hmouz and A. Freeman, "Learning on location: An adaptive mobile learning content framework," in Proceedings of the 2010 IEEE International Symposium on Technology and Society (ISTAS 2010), 2010, pp. 450-456.

[10] A. K. Dey and G. D. Abowd, "Towards a Better Understanding of Context and Context-Awareness," in Proceedings of the 1st international symposium on Handheld and Ubiquitous Computing (HUC '99), 1999.

[11] L. F. Motiwalla, "Mobile learning: A framework and evaluation," Computers \& Education, vol. 49, no. 3, pp. 581-596, Nov. 2007.

[12] D. Furio, S. Gonzalez-Gancedo, M.-C. Juan, I. Segui, and N. Rando, "Evaluation of learning outcomes using an educational iPhone game vs traditional game," Computers \& Education, vol. 64, pp. 1-23, May 2013.

[13] G. Clough, "Geolearners: Location-Based Informal Learning with Mobile and Social Technologies," IEEE Transactions on Learning Technologies, vol. 3, no. 1, pp. 33-44, Jan. 2010.

[14] M. Berry and C. Hodgson, Adventure Education: An Introduction. Routledge, 2011.

[15] A. Doering, “Adventure Learning: Transformative hybrid online education," Distance Education, vol. 27, no. 2, pp. 197-215, Aug. 2006.

[16] A. Doering and J. Henrickson, "Why adventure works in (technology-enhanced) education," in Proceedings of the 7th International Technology, Education and Development Conference (INTED 2013), 2013.

[17] S. Deterding, R. Khaled, L. E. Nacke, and D. Dixon, "Gamification: Toward a Definition," in CHI 2011 Gamification Workshop Proceedings, 2011.

[18] K. M. Kapp, The Gamification of Learning and Instruction: Gamebased Methods and Strategies for Training and Education. Pfeiffer, 2012.

[19] P. R. Polsani, "Use and Abuse of Reusable Learning Objects," Journal of Digital Information, vol. 3, no. 4, pp. 1-5, 2003.

[20] IEEE LTSC, "Draft Standard for Learning Object Metadata," 2002.

[21] “Advanced Distributed Learning (ADL), SCORM 2004 4th Edition," 2004. [Online]. Available: http://www.adlnet.gov/capabilities/scorm/scorm-2004-4th. 